\title{
The radiological, osteodesitometrical and inflammatory parameters aspects in experimentally induced chronic arthritis
}

\author{
Mihaiela Chicu$^{1}$, Oana Ciureanu${ }^{1}$, Gabriela Dogaru² \\ 1. University of Medicine and Pharmacy "Gr. T. Popa "Iasi \\ 2. University of Medicine and Pharmacy "Iuliu Hatieganu" Cluj Napoca \\ Clinical Rehabilitation Hospital Cluj-Napoca
}

\begin{abstract}
Introduction. Chronic inflammatory rheumatic diseases currently comprise over 250 diseases mainly affecting young people and are accompanied by a high degree of physical and mental disabilities.Despite numerous attempts to identify the etiology, they still remain unknown. They were incriminated many factors: genetic, environmental, endocrinological, infectious without specifying exactly what agent could trigger the disease. In the evolution of joint destruction were highlighted three stages: inflammatory synovitis, osteochondral destruction and fibrosis.Methods: Based on these preliminary data, the paper has proposed to make an experimental model of chronic arthritis in laboratory animals - rats Wistar, young and adults and determine the dynamic evolution of radiographic, osteodensitometry and inflammatory parameters.Results. Young animals have presented higher radiological scores compare with the adultes; parameters of bone densitometry (T-score and Z, DEXA, BMD) at the beginning and at the end of the experiment indicated a higher level of bone destruction in group of young animals compared to the adult, while inflammatory parameters (fibrinogen, cervuloplasmina, uric acid) were equal for both groups of animals.Conclusions. Osteodensitometrical, radiological and laboratory parameters constitute the basic indicators in assessing inflammatory diseases, highlighting the intensity of the distructive osteo-articular phenomena.
\end{abstract}

Keywords: arthritis, DEXA, RX.

\section{Introduction}

Radiological parameters (radiological scores Sharp, Van der Heyde, Larsen) used in the quantitative assessment of bone and cartilage destruction in RA were adapted to an experimental model of arthritis in laboratory animals - being used rats of the Wistar, young and adult female, considering that inflammatory diseases mainly affects female subjects and have a more severe evolution in young compared to adult patients.

Osteodensitometry parameters: DEXA, respective $\mathrm{T}$ and $\mathrm{Z}$ scores, BMD (Bone Mineral Density) they also can identify the changes in bone mineral density, bone loss being correlated with the local synovitis.
Inflammatory parameters were determined in the dynamic throughout the experiment.

Material and method. The experimental study was conducted over a period of eight weeks, on Wistar rats young and adult female, which was induced arthritis by injecting tibial-femoral joint with $1 \%$ carrageenan, $0.02 \mathrm{ml}, 3$ times/week. The animals received standard food and were properly hydrated. During the experiment, the fibrinogen, cervuloplasmin and uric acid levels were determined in the dynamic. Radiological examination was conducted in Semiologically and Radiology Clinic (leader prof. dr. Vasile Vulpe) of University of Agricultural Sciences and Veterinary Medicine "Ion Ionescu de la Brad" Iasi, and the bone densitometry aat the Endocrinology Clinic 
of University Hospital „St. Spiridon” Iasi using Helicon 100 (made in USA).

Results. Regarding on Larsen radiological scores valid to human (0 to 5 ), we have tried to develop similar scores in laboratory animals as follows: score 0 normal aspect of the tibial-femoral joint; score 1 - 25\% destruction; score 2 - 50\% destruction; score 3 - stiffen marrow.

\section{Bone densitometry examination}

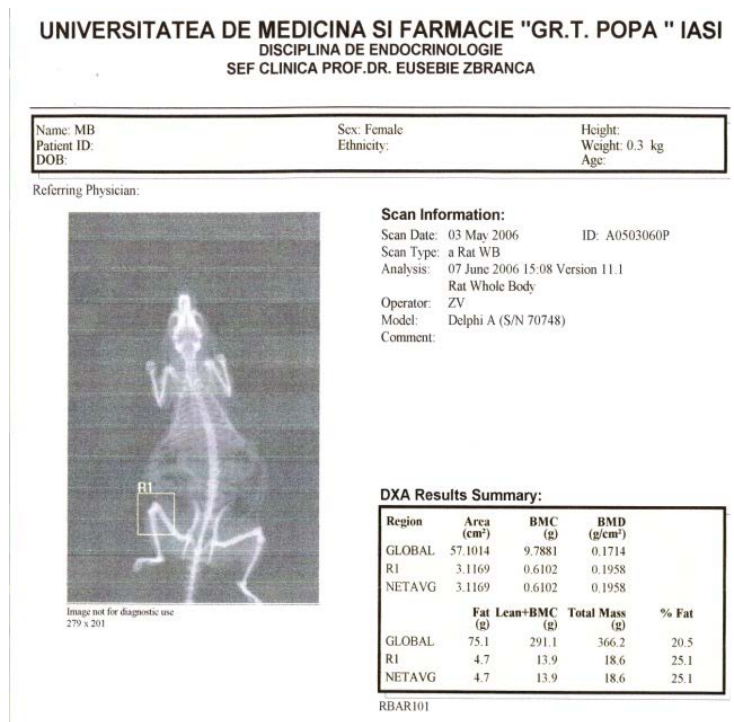

Young rat witness

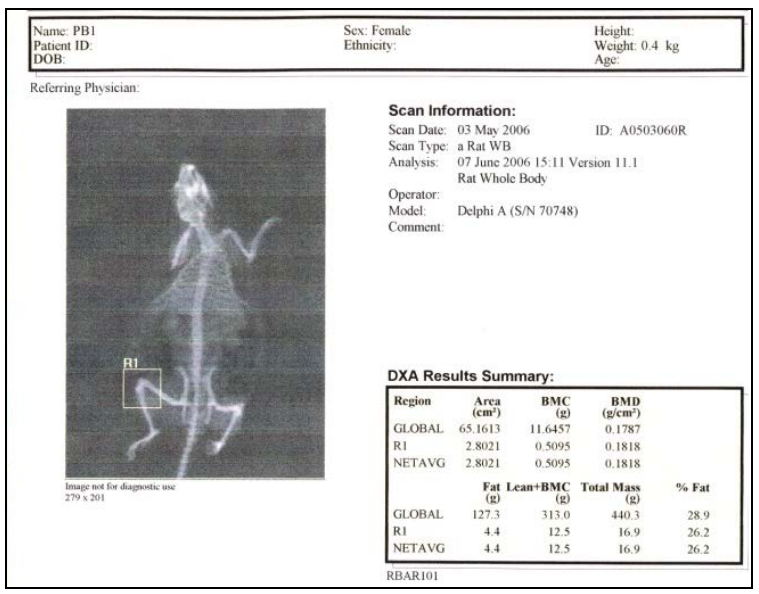

Adult rat with arthritis (may investigation) 


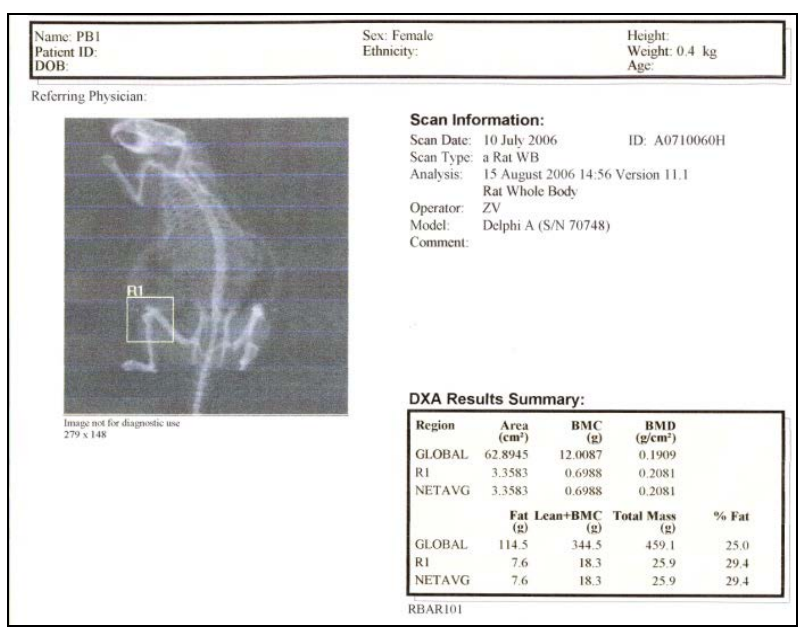

Adult rat with arthritis (july investigation)

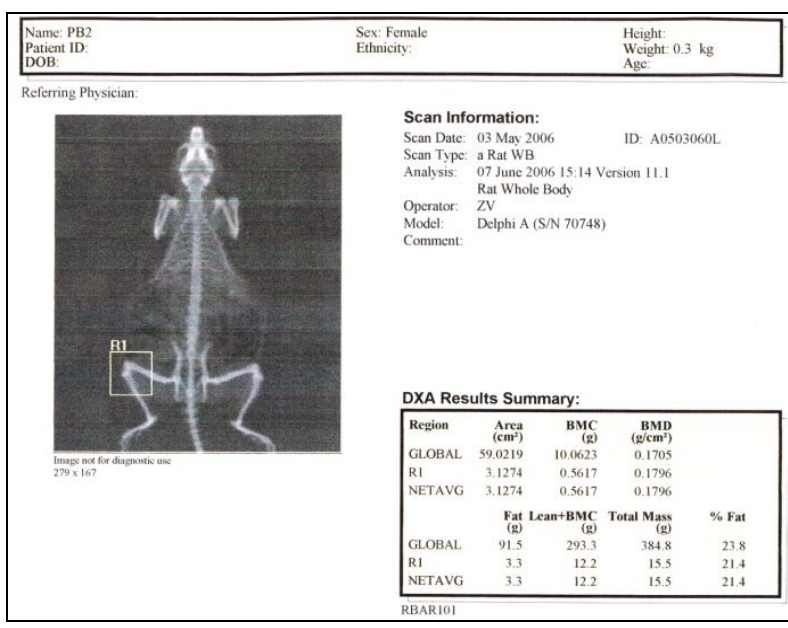

Young rat with arthritis (may investigation)

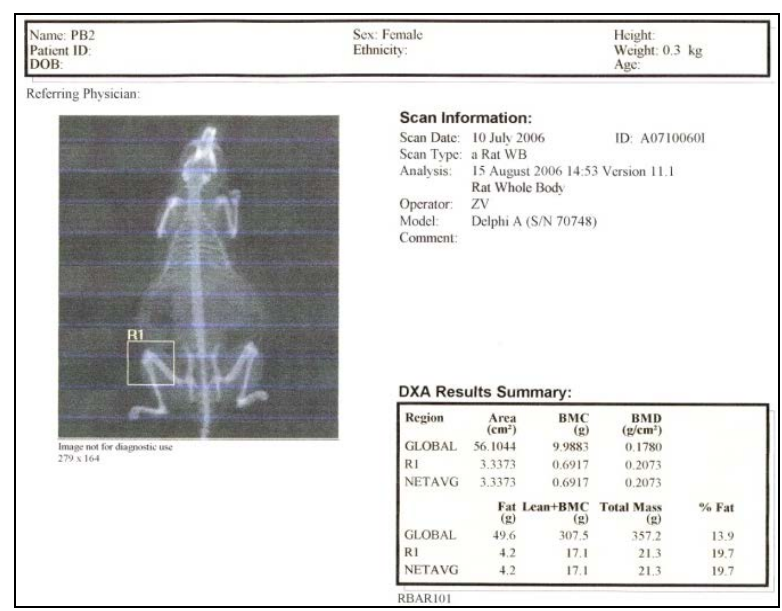

Young rat with arthritis (july investigation) 
Inflammatory parameters in young animals

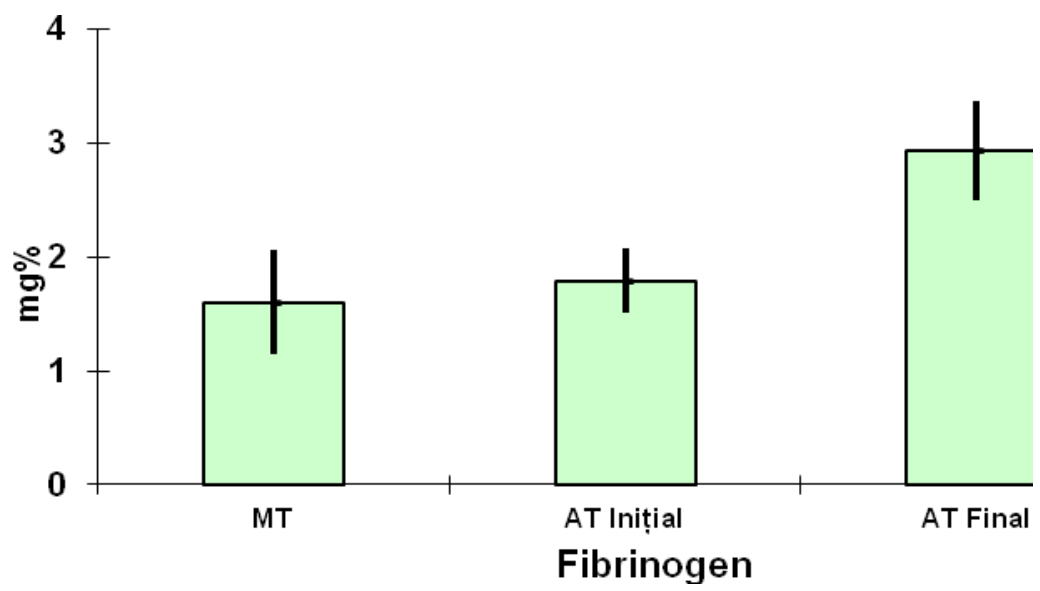

Average values of fibrinogen in young rats group with arthritis comparing to witnewss group

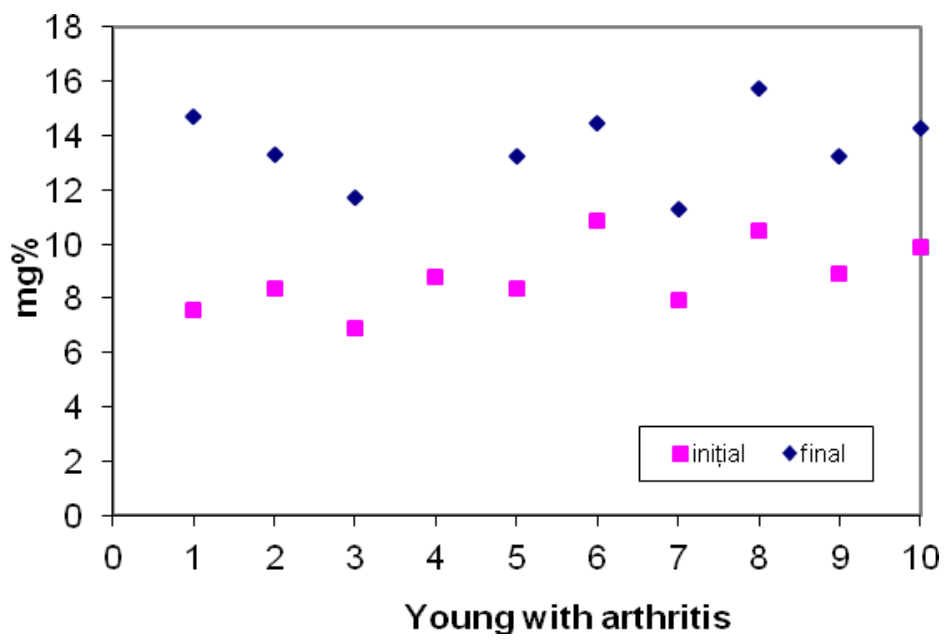

Ceruloplasmin levels variation in young rats group with arthritis

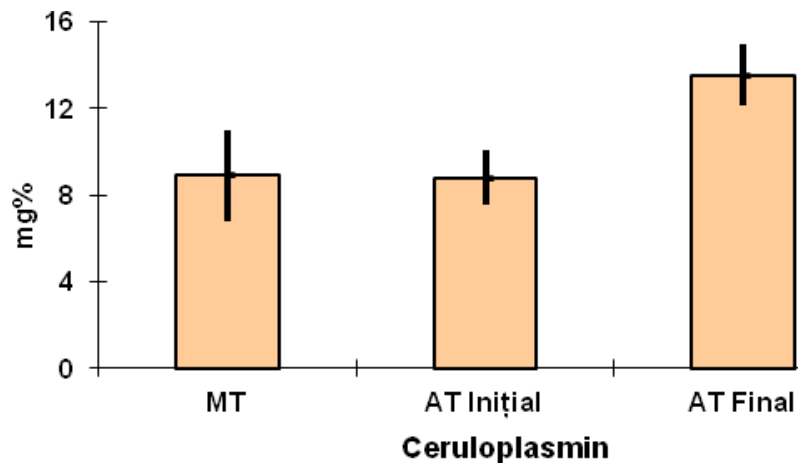

The average values of ceruloplasmin in young rats group with arthritis comparing to witness group 


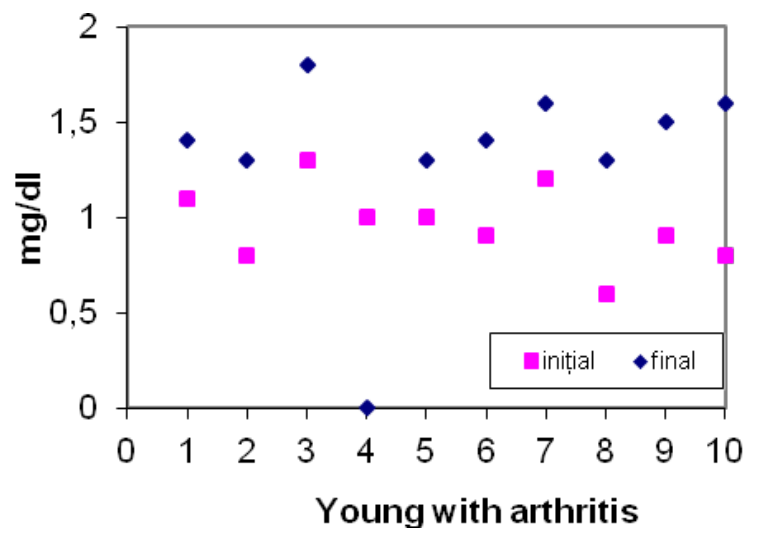

The variation of uric acid levels in young rats group with arthritis

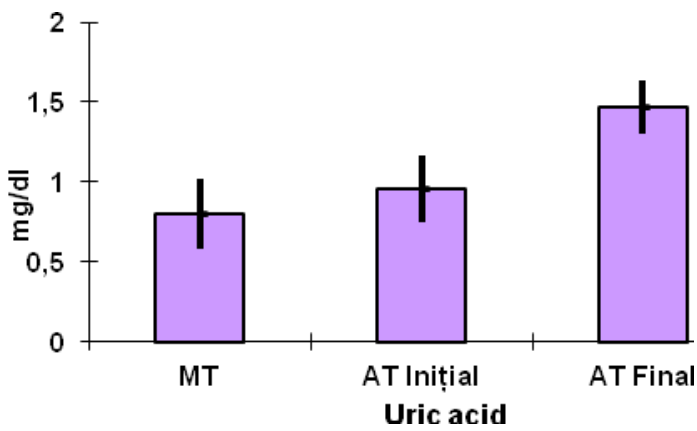

Average values of uric acid in young rats group comparing to witness group

\section{Inflammatory parameters in adult animals}

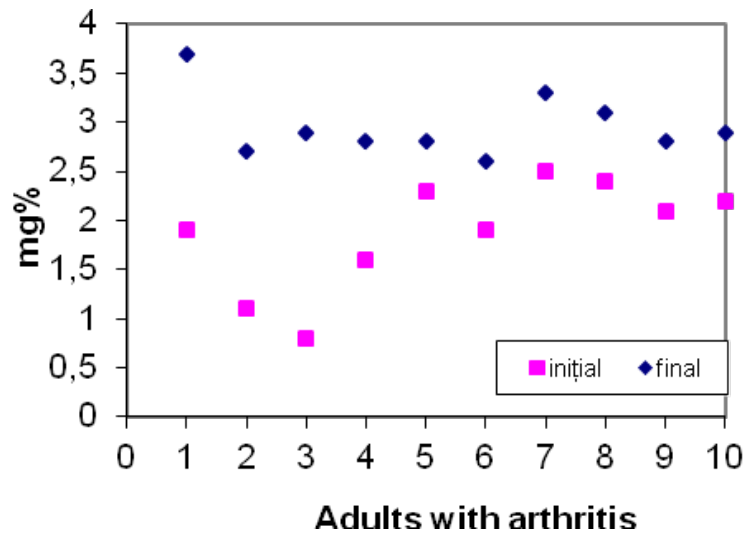

The variation of fibrinogen levels in adults rats group with arthritis 


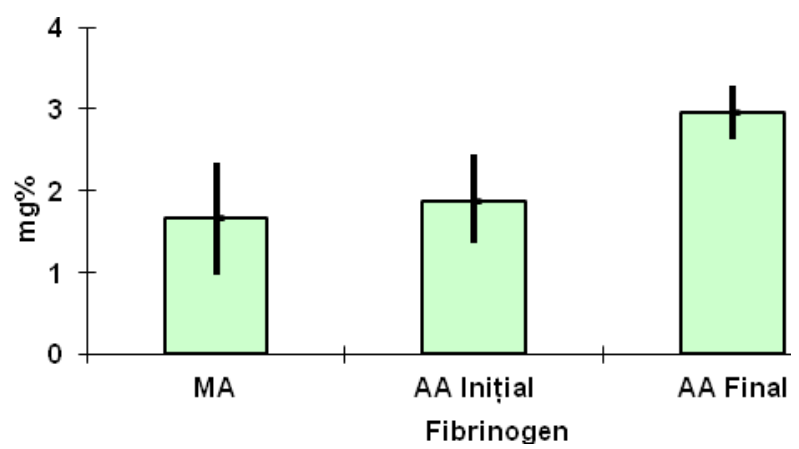

Average values of fibrinogen in adults tars group comparing to witness group

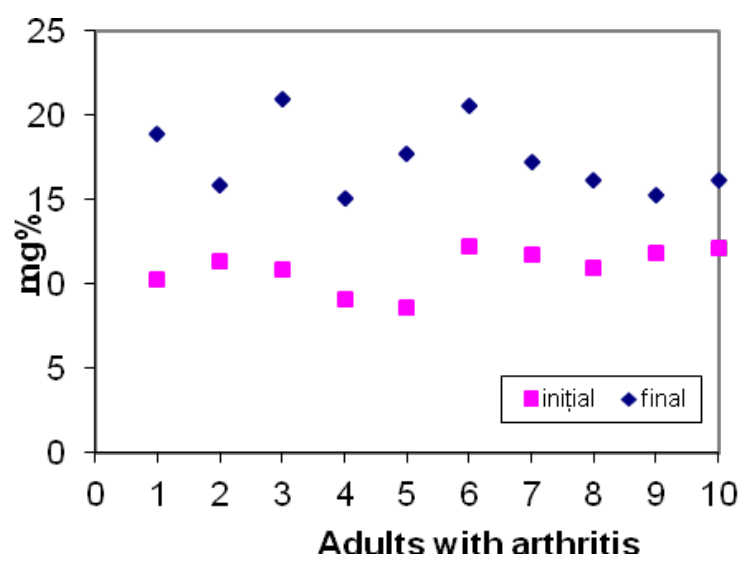

The variation of ceruloplasmin levels in adults rats group with arthritis

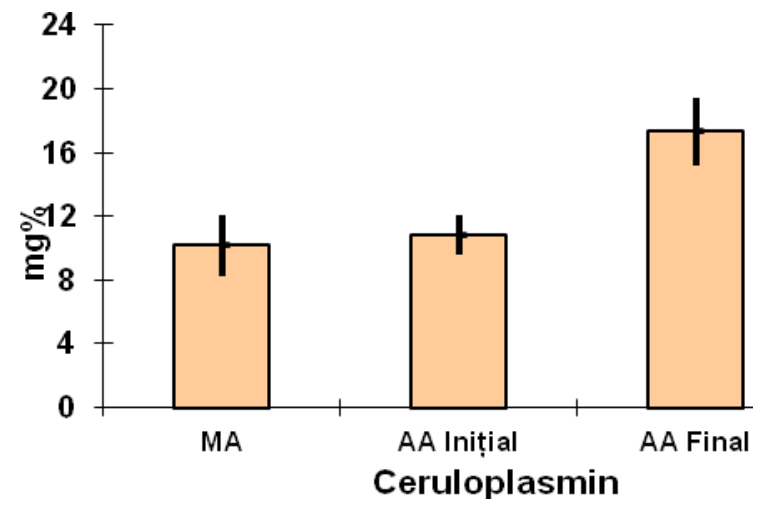

The average ceruoplasmin values in adults rats group with arthritis comparing to witness group 


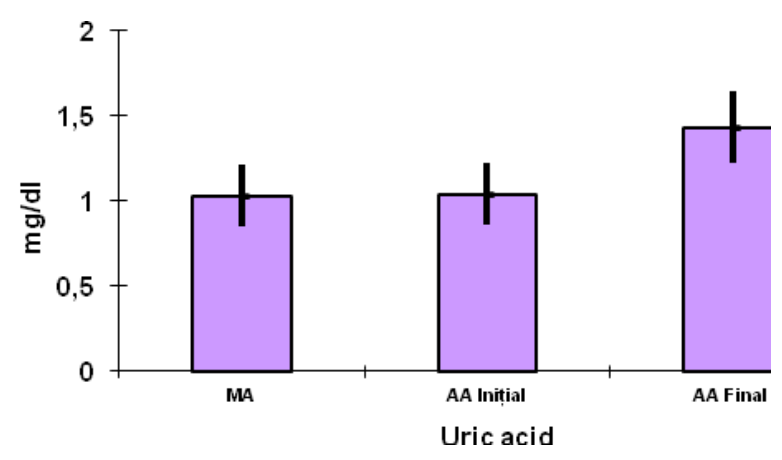

Average uric acid values in adults rats group comparing to witness group

Conclusions. Intensity of bones and cartilage destruction phenomena was higher in the young animals compared to adult ones, thus confirming the data presented in the literature.

\section{Bibliography}

Kellgren JH, Lawrence JS: Radiological assessment of rheumatoid arthritis. Ann. Rheum Dis 1997, 16:485-493;

Larsen A, Dale K, Eek M: Radiographic evaluation of rheumatoid arthritis and related conditions by standard reference films. Acta Radiol Diag 1977, 18:481-491; Lassere M, Houssein D, Scott D, Edmonds J: Reference curves of radiographicdamage in patients with rheumatoid arthritis: application of quantile regression and fractional polynomials. J rheumatol 1977, 24:128894;

Lewis EJ, Sedgwick AD, Hanahoe THP: Automatic quantification of rat plasma acute phase protein levels reactants in experimental inflamation. J. Pharmacol Methods 1989; 21:183-94. 